\title{
A case of papular elastorrhexis
}

\author{
Meng Jiang, Wenbo Bu, Xu Chen, Heng Gu
}

Institute of Dermatology, Jiangsu Key Laboratory of Molecular Biology for Skin Diseases and STIs, Chinese Academy of Medical Science and Peking Union Medical College, Nanjing, China

An 11-year-old girl presented with a 2-year history of flesh-colored papules on her extremities (Figure 1). The lesions were asymptomatic, of oval or irregular shape and increased gradually both in size and numbers without any treatment. She denied a history of any other inflammatory dermatoses or trauma on the involved area. There was no family history of similar lesions. Dermatological examination showed that multiple flesh-colored, non-follicular papules were discrete and distributed over her lower limbs. The lesions were approximately 5-10 mm in diameter, of various shapes and slightly hard. No other primary or secondary lesions (erythema or scales, etc.) can be seen around the involved area. Histopathological examination of the skin lesion on the left thigh revealed hyperkeratosis, slight perivascular lymphocytes infiltration and a mild hyperplasia of collagen fiber in the dermis. Elastic tissue staining (Resorcinol-Fuchsin stain) showed a significant reduction, fragmentation or complete loss of elastic tissue in upper and mid dermis (Figure 2). Based on the clinical and histopathological features, a diagnosis of papular elastorrhexis was made. No treatment was performed because of the benign process of this disease and asymptomatic character of the lesions. She was followed up for one year and showed no improvement in the involved lesions.

Papular elastorrhexis (PE) is a rare acquired elastic tissue disorder which was first described by Bordas et al. [1] in 1987. The etiology of this disease is still unclear. Papular elastorrhexis usually occurs in children or adolescents with a predilection for women. Its clinical characteristics are multiple asymptomatic, non-follicular flesh-colored papules, between 3 and $5 \mathrm{~mm}$ in size. Papules are firm and mainly distributed over the trunk or upper extremities with no fusion. In addition, there is no osteopoikilosis or similar family history and usually occurs without any previous history of acne, inflammation or trauma. The prominent histopathological feature of PE is the reduction, fragmentation or complete loss of dermal elastic fibers. Collagen fibers can be normal, focally thickened or even homogenized with a few lymphocytes infiltrated around the blood vessels [2].

Papular elastorrhexis has not received a well-recognized division and it is still controversial whether PE is a distinct entity or a variant of nevus anelasticus (NA) or abortive form of Buschke-Ollendorff syndrome (BOS) $[1,3]$. But it is generally confirmed that PE is a distinct entity different from other elastic tissue disorders including NA, BOS, eruptive collagenoma, white fibrous papulosis of the neck, pseudoxanthoma elasticum-like papillary dermal elastolysis (PXE-PDE), mid-dermal elastolysis and papular acne scars $[4,5]$. These diseases clinically present with scattered white-to-yellow papules and show a reduction or loss of dermal elastic tissue in histology. Differential diagnosis of PE can be established with other

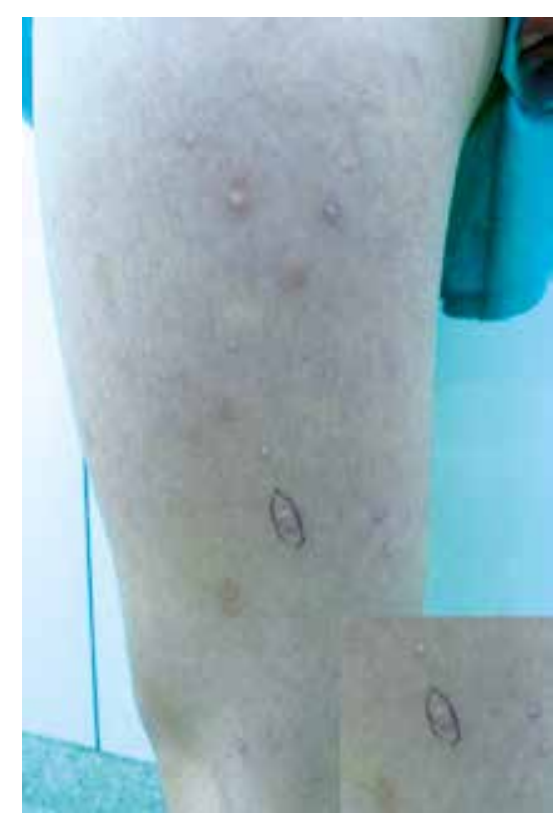

Figure 1. Multiple flesh-colored non-follicular papules scattered on lower limbs

Address for correspondence: Xu Chen, Bu Wenbo, Heng Gu, Institute of Dermatology, Jiangsu Key Laboratory of Molecular Biology for Skin Diseases and STIs, Chinese Academy of Medical Science and Peking Union Medical College, 12 Jiangwangmiao St, Nanjing 210042, China, phone: +86 25 8547 8919, fax: +86 25 85414477, e-mail: doctor_chx@hotmail.com, buwenbo@163.com, doctor_guheng@hotmail.com Received: 12.08 .2017 , accepted: 19.10 .2017$. 

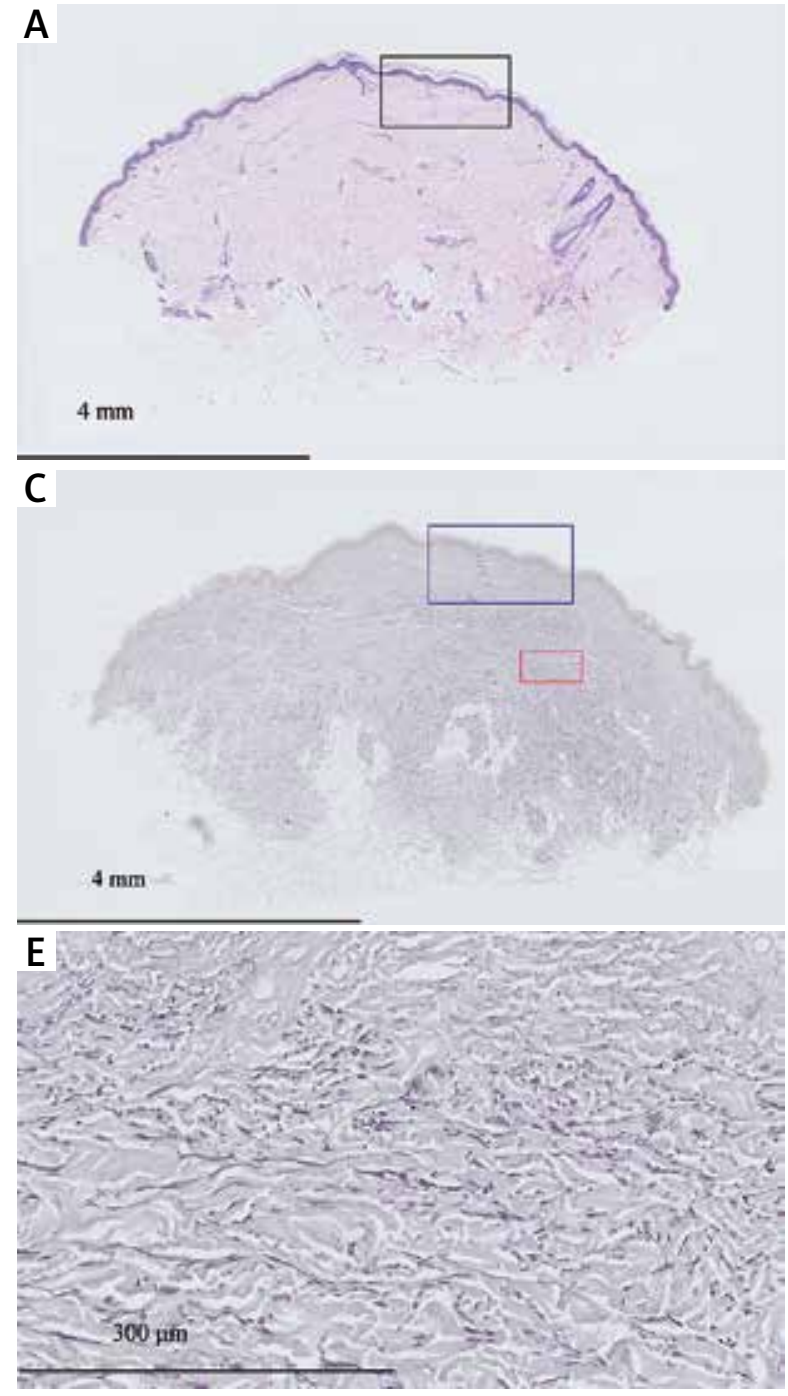

elastic fiber disorders according to the different clinical features and primary histopathological changes. In our case, the onset age, the absence of osteopoikilosis, no family history or trauma history, clinical features of the papules, and the histopathological features of focal fragmentation and loss of elastic fibers are in accord with the character of PE. However, there is no recommended treatment for PE, including topical tretinoin, oral antibiotics or isotretinoin have been proved invalid. Notably, several cases show improvement after the intralesional injection of triamcinolone $[4,6]$. In view of the benign process of PE, we suggest a long-term follow-up observation if possible.

\section{Acknowledgments}

$\mathrm{Xu}$ Chen and Wenbo Bu are supported by the PUMC Youth Fund and Fundamental Research Funds for the Central Universities (3332015116, 3332014008 , 2016RC320005 and 2016ZX320014).
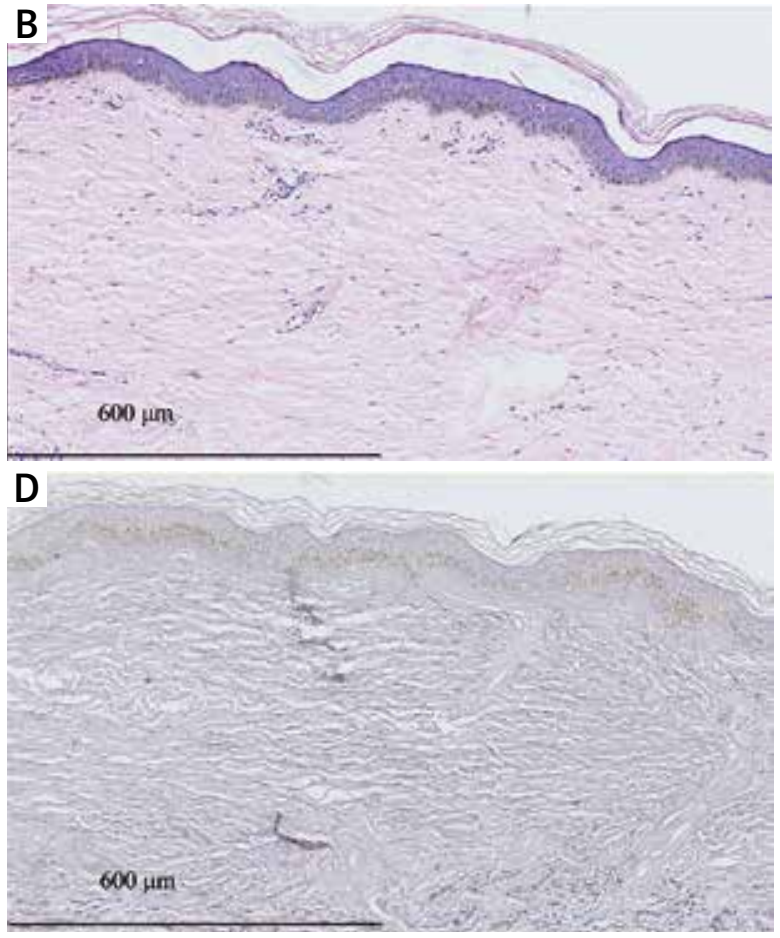

Figure 2. Histopathological examination showed hyperkeratosis, perivascular slight lymphocytes infiltration and a mild hyperplasia of collagen fiber in the dermis $(H+E$, $A-1.5 \times ; B-10 x)$. Elastic tissue staining showed a significant reduction and fragmentation of elastic tissue in the dermis (Resorcinol-Fuchsin stain, C - 1.5x; D - 10x; E - 20x)

\section{Conflict of interest}

The authors declare no conflict of interest.

\section{References}

1. Bordas X, Ferrandiz C, Ribera M, et al. Papular elastorrhexis: a variety of nevus anelasticus? Arch Dermatol 1987; 123: $433-4$.

2. Buechner SA, Itin P. Papular elastorrhexis. Report of five cases. Dermatology 2002; 205: 198-200.

3. Schirren H, Schirren CG, Stolz W, et al. Papular elastorrhexis: a variant of dermatofibrosis lenticularis disseminata (Buschke-Ollendorff syndrome)? Dermatology 1994; 189: 368-72.

4. Lewis KG, Bercovitch L, Dill SW, et al. Acquired disorders of elastic tissue: Part II. decreased elastic tissue. J Am Acad Dermatol 2004; 51: 165-85; quiz 186-168.

5. Luo DQ, Liu JH, Chen MC, et al. Papular elastorrhexis: report of four cases and review of literature. Arch Dermatol Res 2015; 307: 99-108.

6. Lee SH, Park SH, Song KY, et al. Papular elastorrhexis in childhood improved by intralesional injections of triamcinolone. J Dermatol 2001; 28: 569-71. 